\title{
CORRESPONDANCE ENTRE DEUX SURFACES PAR TROIS COUPLES DE TANGENTES PARALLÈLES
}

\author{
PAVEL DRĂGILĂ
}

1. En continuant nos recherches sur la correspondance de deux surfaces, par parallélisme des plans tangents homologues, nous avons établi dans le travail précedent [2] que, en dehors du cas de surfaces se correspondant par parallélisme des deux directions conjuguées, étudié par Peterson [1, p. 24], il y a encore des couples de deux surfaces, qui se correspondent par parallélisme des plans tangents et par parallélisme d'un seul système de tangentes. Nous avons montré encore qu'on peut déterminer effectivement des couples de telles surfaces par l'intégration du système

$$
\begin{aligned}
& \bar{r}_{u}=r_{u}-r_{v}, \\
& \bar{r}_{v}=r_{v},
\end{aligned}
$$

ou bien du l'autre système

$$
\begin{aligned}
& \bar{r}_{u}=r_{u}-r_{v}, \\
& \bar{r}_{v}=k r_{u},
\end{aligned}
$$

$k$ étant une constante, qui satisfasse la condition

$$
k>1 / 4 \text {. }
$$

2. Nous nous sommes proposé d'étudier actuellement la correspondance des deux surfaces $S, \bar{S}$, définies par le système (2), dans le cas où la constante $k$ vérifie l'inégalité contraire

$$
k<1 / 4 \text {. }
$$

Dans les calculs qui suivent nous désignons, comme d'habitude, les deux surfaces par $S, \bar{S}$, et nous représentons par les notations vectorielles $r, r_{u}, r_{v}, r_{u v}, \cdots$ les expressions des coordonnées $x, \cdots$ et des leurs dérivées $\partial x / \partial u, \cdots, \partial x / \partial v, \cdots, \partial^{2} x / \partial u \partial v, \cdots$.

Cherchons maintenant à faire un changement de variables

$$
u=\phi(s, t), \quad v=\psi(s, t),
$$

de manière que les nouveaux vecteurs $r_{s}, r_{t}$, sur la première surface soient parallèles aux vecteurs correspondants $\bar{r}_{t}, \bar{r}_{s}$, sur la seconde surface. En posant les conditions de parallélisme

Received by the editors March 26, 1958. 


$$
\frac{\bar{x}_{s}}{x_{t}}=\frac{\bar{y}_{s}}{y_{t}}=\frac{\bar{z}_{s}}{z_{t}}, \quad \frac{\bar{x}_{t}}{x_{s}}=\frac{\bar{y}_{t}}{y_{s}}=\frac{\bar{z}_{t}}{z_{s}}
$$

et en faisant ensuite les substitutions

$$
\bar{r}_{s}=\left(r_{u}-r_{v}\right) u_{s}+k r_{u} v_{s}, \quad \bar{r}_{t}=\left(r_{u}-r_{v}\right) u_{t}+k r_{u} v_{t},
$$

nous obtenons les deux équations

$$
\begin{aligned}
& u_{s} u_{t}+u_{s} v_{t}+k v_{s} v_{t}=0, \\
& u_{s} u_{t}+v_{s} u_{t}+k v_{s} v_{t}=0,
\end{aligned}
$$

d'où il résulte

$$
u_{s} v_{t}-v_{s} u_{t}=0 .
$$

qui est manifestement impossible.

En écrivant l'autre système de relations

$$
\frac{\bar{x}_{s}}{x_{s}}=\frac{\bar{y}_{s}}{y_{s}}=\frac{\bar{z}_{s}}{z_{s}}, \quad \frac{\bar{x}_{t}}{x_{t}}=\frac{\bar{y}_{t}}{y_{t}}=\frac{\bar{z}_{t}}{z_{t}},
$$

et en effectuant les mêmes calculs, nous trouvons les deux équations

$$
\begin{aligned}
& u_{s}^{2}+u_{s} v_{s}+k v_{s}^{2}=0, \\
& u_{t}^{2}+u_{t} v_{t}+k v_{t}^{2}=0,
\end{aligned}
$$

qui sont compatibles, dans le cas quand l'inégalité (3) est satisfaite.

Si nous notions $k_{1}, k_{2}$ les racines réelles et distinctes de l'équation

$$
p^{2}+p+k=0
$$

il est aisé de voir que nous pourrions faire le changement de variables

$$
u=k_{1} s+k_{2} t, \quad v=s+t .
$$

Nous avons découvert ainsi un nouveau cas, le plus curieux peutêtre, de correspondance par trois couples de tangentes parallèles.

3. Il est aisé de déterminer des couples de telles surfaces. Par l'intégration du système particulier

$$
\begin{aligned}
& \bar{r}_{u}=r_{u}-r_{v}, \\
& \bar{r}_{v}=-2 r_{u}
\end{aligned}
$$

nous avons obtenu les paraboloïdes

$$
\begin{array}{ll}
x=u, & \bar{x}=u-2 v, \\
y=v, & \bar{y}=-u, \\
z=u^{2}+2 v^{2}, & \bar{z}=u^{2}-4 u v .
\end{array}
$$


Faisant le changement de variables

$$
u=s-2 t, \quad v=s+t,
$$

nous trouvons les nouvelles expressions des coordonnées

$$
\begin{array}{ll}
x=s-2 t, & \bar{x}=-s-4 t, \\
y=s+t, & \bar{y}=-s+2 t, \\
z=3 s^{2}+6 t^{2}, & \bar{z}=-3 s^{2}+12 t^{2},
\end{array}
$$

qui sont liées par les relations

$$
\begin{aligned}
& \bar{r}_{s}=-r_{s}, \\
& \bar{r}_{t}=2 r_{t} .
\end{aligned}
$$

4. Nous avons poursuivit, dans ce travail, en premier lieu, de prouver l'existence des correspondances par trois couples de tangentes parallèles, et, en second lieu, de déterminer effectivement de telles couples de surfaces. On pourrait considérer aussi les systèmes plus généraux, à coefficients variables

$$
\begin{aligned}
& \bar{r}_{u}=a r_{u}+b r_{v}, \\
& \bar{r}_{v}=c r_{u},
\end{aligned}
$$

mais nous n'avons pas réussi de donner, jusqu'à present, l'interprétation géométrique satisfaissante. Cependant il y a lieu de remarquer un fait géométrique intéressant. Nos considérations restent toutes valables encore dans le cas quand le coefficient $k$ du système (2) est une fonction arbitraire des paramètres $u$, v. Dans ce cas il y a des regions, sur les deux surfaces, définies par la relation

$$
k(u, v)>1 / 4,
$$

dans lesquelles on aura une correspondance par un seul système de tangentes parallèles, et dans les autres regions une correspondance par trois systèmes de tangentes parallèles. Pour distinguer cet espéce curieuse de correspondance nous l'appellerons polymorphe, par opposition au cas ordinaire, quand le nombre de couples de tangentes parallèles est partout le même, que nous l'appellerons isomorphe.

En conclusion, la correspondance définie par un système d'équations (2) à coefficients constants est toujours isomorphe, tandis que cela définie par un système à coefficients variables peut etre isomorphe ou polymorphe. Dans ce dernier cas il n'est pas possible de trouver effectivement le changement de variables (4).

5. A la fin nous croyons utile d'attraire l'attention sur la nécéssité 
d'étudier la correspondance des deux surfaces par trois, et eventuellement par quatre couples de tangentes, aussi au point de vue projectif. En effet nous savons déjà qu'il est possible d'obtenir des couples de deux surfaces se correspondant par trois et aussi par quatre couples de tangentes incidentes, les droites déterminées par ces points d'incidence restant coplanaires. Il se pose tout naturellement le nouveau problème de chercher s'ils existent des couples de surfaces, telles que les droites déterminées par les trois, respectivement quatre, points d'incidence, constituent des congruences. Jusqu'à présent furent étudiées seulement les congruences de droites, déterminées par deux points d'incidence, par plusieurs géomètres, parmi lesquels nous pouvons citer: G. Fubini, G. Tzitzeica, A. Pantazi, Ms. V. Grove, S. Finikof, L. Godeaux, G. Gheorghiu, I. Mihăilescu, M-me M. Bica.

\section{BibliogRAPHIE}

1. K. M. Peterson, Sur les relations et les affinités entre les surfaces courbes, Ann. Fac. Sci. Univ. Toulouse, 1905.

2. P. Drăgilă, Correspondances singulières par parallelisme des plans tangents des deux surfaces, Proc. Amer. Math. Soc. vol. 9 (1958) pp. 518-521.

Timisoara, Roumania 